\title{
A Nurse's Guide to Nuclear Medicine
}

Aims:

1. To enable nurses to give their patients an informed overview of what they might expect when having common nuclear medicine examinations.

2. To heighten nurse awareness about practical radiation protection issues that might need to be addressed within their normal working routine.

\section{Intended learning outcomes:}

1 For some common nuclear medicine imaging procedures, explain what a typical patient experience might consist of.

2 State some reasons why patients might need to be referred for some common nuclear medicine procedures and indicate what value the results may have for their management.

3 List typical radiation protection issues which might arise from patients who have had a recent nuclear medicine imaging procedure.

\section{Introduction}

Nurses play invaluable roles in the care, diagnosis and treatment of patients. Within all of these roles there is an expectation that nurses should be able to give informed explanations about diagnostic procedures that their patients may 
undergo. Increasing patient understanding can improve decision making for informed choice and reduce their anxiety; it can also heighten procedural compliance, improve procedure quality and outcome and enhance patient experience (NHS Institute for Innovation and Improvement, 2008; Department of Health, 2010; MHRA, 2012). Taken from a patient's perspective this article explains how nuclear medicine procedures are performed, thereby helping nurses answer patients' questions about what some common nuclear medicine imaging procedures might involve. The article also includes some information about radiation protection because nurses will come across situations which involve radioactive patients - a consequence of nuclear medicine procedures. The article intentionally omits detailed technical information about nuclear medicine because that tends only to be important to those who work in the nuclear medicine department. However at the end of the article there are some suggestions for further reading for those who might be interested in it.

\section{Time out 1}

If you were a patient and you had just been told that your doctor was going to send you to the nuclear medicine department for a bone scan what would be going through your mind? Write down what you think might happen to you. List your worries and any questions that you would like to ask.

\section{Time out 2}

Watch this online video of a patient attending for a nuclear medicine bone scan (https://www.youtube.com/watch?v=AfppkSYFgYg). Has 
this answered any of your worries from time out 1 or created new ones? If it has created new ones please write them down as well.

\section{What is nuclear medicine?}

The name 'nuclear medicine' may sound frightening to some, but it simply comes from the fact that it is a branch of medicine that employs nuclear radiation - natural emissions from the nucleus of radioactive atoms. Nuclear medicine is mostly concerned with diagnostic imaging and although in large hospitals it can be a department in its own right, in others it may be included as part of a radiology or a medical physics department. It may sometimes go under other names such as 'radioisotope scanning' or 'gamma camera unit'. Nuclear medicine can also be used for treatment of some conditions, although that is beyond the scope of this article.

Nuclear medicine uses radioactive substances (radiopharmaceuticals) to identify or exclude pathology. A radiopharmaceutical is a chemical (which determines where it goes to in the body) attached to a radioactive label (which allows it to be detected externally). Radiopharmaceuticals can be administered to patients in several ways, including intravenous injection, inhalation and ingestion. Pathology is demonstrated by the site and amount of radiopharmaceutical which accumulates in specific areas within the body. The radioactive label is carefully chosen so that it decays away quite rapidly (usually with a half-life of a few hours). The radioactive label emits radiation which can be detected by a scanner to make an image of where the 
radiopharmaceutical has accumulated in the patient. Examples of nuclear medicine scanner technologies include the gamma camera (Figure 1) and the Positron Emission Tomography (PET) scanner (Figure 2). The nuclear medicine images can be used to assess the amount of radiopharmaceutical taken up into a specific pathological site, which can be valuable for assessing the severity of any pathology. Nuclear medicine uses a wide range of different radiopharmaceuticals and each has specific targeting properties for different organs or physiological pathways.

Two pathological characteristics can be imaged - anatomy and physiology. Xray technologies (including computed tomography (CT)), ultrasound and magnetic resonance imaging (MRI) continue to serve us well for imaging anatomy. Relatively recently advances in ultrasound and magnetic resonance have enabled physiology to be imaged and even more recently optical imaging has started to show promise in this regard too. However, since its inception, nuclear medicine has been the largest contributor to physiological imaging. Imaging physiology has many values, including its ability to detect pathology earlier than anatomical imaging in some instances (Brenner et al, 2012). When assessing pathology it is often necessary to image physiology as well as anatomy, so that a full understanding can be reached about the disease and how it might be managed. Consequently it would not be unusual for a patient to have a range of diagnostic imaging procedures conducted using several imaging modalities (eg ultrasound, MRI, nuclear medicine and X-ray). 
Although X-rays and nuclear medicine both involve the use of ionising radiation to produce diagnostic images they differ in the way that the images are taken and so the patient experiences are different. X-ray images are taken by shining a short burst of X-rays through the patient to produce a shadow of their internal organs. Images can be acquired within a few seconds and the radiation is only present whilst the X-rays are on. In contrast nuclear medicine images are taken by detecting the radiation coming out of the patient and because very small amounts of radioactivity are used images can take several minutes to acquire. The radiation may also remain in the patient for several hours after the test is finished.

As noted above, radiopharmaceuticals are radioactive substances. Radioactive substances are unstable and so they emit energy in the form of ionising radiation to become stable. This ionising radiation can be in the form of particles (eg beta particles) which are easily stopped and so get absorbed in the patient or electromagnetic waves (eg gamma rays) which mostly emerge out of the patient. The radiopharmaceuticals used in diagnostic nuclear medicine tend to emit gamma rays since these can be detected from outside the patient. Ionising radiation can be hazardous and because of this its use in medicine is regulated in law (IRR, 1999, IRMER, 2000). However the radiation dose that a patient receives from a diagnostic nuclear medicine procedure is very small and is about the same as the unavoidable amount of radiation that everyone receives from natural background radiation each year. Nevertheless, for the protection of patients, a firm clinical justification that the benefit will outweigh the risk must be given before a procedure using ionising radiation is 
conducted. This justification will be done by nuclear medicine staff who understand the risks involved, but sufficient clinical information about the patient must be provided by the referring doctor in order for them to assess the potential benefit. If this cannot be provided then the procedure should not be done. Also, for the protection of the patient, the procedure itself should use the least amount of radiation possible.

\section{Time out 3}

If you work in the NHS you can get free access to some useful online learning resources created by e-Learning for Healthcare (http://portal.e-lfh.org.uk/Register). After registering, from the list of available programmes put a tick against 'Interpretation of Radiological Images' which is in the 'Allied Health Professionals' group and save the changes. When you have been granted access, log on to the e-LfH portal (http://portal.e-lfh.org.uk/Account/logon) and from 'My eLearning' select 'Interpretation of Radiological Images'. Scroll down to find 'Nuclear Medicine' and review session 1 (Principles of Nuclear Medicine).

Now reflect on the list you wrote in time out 1 and time out 2 . Has the introduction in this article and the e- $\mathrm{LfH}$ session on the principles of nuclear medicine answered any of your worries and questions? 


\section{What happens during nuclear medicine procedures?}

Sometimes difficulties can arise when the patient has been inadequately informed about the examination that they are about to undergo. The difficulties fall into two categories. Firstly the patient wants to know what might be wrong with them and what the examination results might mean to them. These are very hard questions for nuclear medicine staff to answer because normally they will not know 'what the patient knows' or anything about their emotional and socio-economic circumstances. Certain pathologies, like cancer, have major implications for patients, carers and their families so nuclear medicine staff are extremely cautious when responding. Therefore, if possible, patients should arrive with a clear understanding of why the investigation is being carried out and what the examination outcome might mean to them.

Secondly the patient doesn't know what the examination entails. This should always be addressed by nuclear medicine staff who will give a careful explanation and answer the patient's questions when they arrive in the department. However it is best if patients' worries can be dealt with in advance. Indeed if the patient didn't realise how long the procedure might take it could conflict with their expectations, and lessen their experience. Outpatients are usually sent an explanatory appointment letter, which should address this category of information needs. However, in-patients tend to rely on hospital staff for information. So it is likely that the nurse, or other members of the care team, will play important roles in explaining why their 
patient needs the scan and what the result might mean to them and also what the nuclear medicine procedure involves.

\section{Bone scan}

The bone scan is the most common imaging procedure performed in the nuclear medicine department. As noted in the video (time out 1), the procedure is quite long as it comprises of an injection followed by a waiting period before imaging.

The bone scan produces images that show how rapidly bones are growing - or more accurately how fast they are metabolising phosphate into the bone matrix. The patient is given a small intravenous injection of a radiopharmaceutical containing a phosphate compound that is labelled with a radioactive element called technetium-99m. Then the patient has to wait for between 2 and 4 hours for the radiopharmaceutical to become incorporated into the bones. During this waiting time the patient may return to the ward if they are an in-patient or be allowed to go elsewhere in the hospital or outside if they are an out-patient. The nuclear medicine department will tell the patient if they have any local restrictions on where they may go. After about 3 hours the patient returns to the nuclear medicine department and a gamma camera is used to take pictures which show the distribution of bone metabolism throughout the patient's skeleton (see figure 1). An example of a normal whole body bone scan is shown in figure 3. Normal bone metabolism is shown in shades of grey and any areas of increased bone turnover will appear as black. 
In this example the black area in the patient's pelvis is radiopharmaceutical in their bladder, which is normal. Patients will be asked to empty their bladder just before the images are taken in order to minimise this activity, otherwise it can obscure the pelvic bones. Unless it is contraindicated, patients may be asked to drink plenty of water in order to encourage complete bladder emptying, but otherwise no special preparation is needed before a bone scan. In order to get good pictures, the gamma camera must be placed as close as possible to the patient and they must remain still during the scan. Each picture takes several minutes to acquire and during this time the patient will be asked to lie flat on their back (figure 1), so if they find this difficult it is worth discussing with the nuclear medicine department beforehand. Sometimes it may be possible to acquire several 5 minute images instead of one 20 minute image if the patient finds this easier. The timing of the patient's pain medication may also need adjusting to allow them to cope with the scan.

After the whole body images are taken it is sometimes necessary to acquire additional images using a technique called single photon emission computed tomography (SPECT). For this the gamma camera rotates slowly around the patient taking images as it goes, which takes about 10 minutes. Then a computer reconstructs these images into cross sectional slices across the body. Some modern gamma cameras also have an X-ray CT scanner attached to them and so sometimes further images may be acquired using a CT scan if this is required. This hybrid SPECT-CT scan has the advantage of combining the physiological information from the nuclear medicine SPECT scan with the anatomical information from the CT scan. 
The bone scan is very sensitive for detecting areas of abnormal bone turnover which it can often be detected before there any anatomical changes visible on an X-ray. However it is not able to be very specific about the causes of an abnormality and so it is often used in conjunction with other tests and clinical information. A bone scan is used in some patients with known cancers (for example prostate cancer) for assessing the extent of disease, and investigating bone pain in suspected metastatic tumours. The results can be used for staging and monitoring disease progression and the response to treatment. The finding of whether or not the patient has bony metastases can determine the appropriate treatment and management options. However a bone scan is also indicated in many non-oncological conditions including trauma, orthopaedic disorders, sports injuries, metabolic bone disease, infection, degenerative disease and avascular necrosis. In these cases the result can either support the initial diagnosis or maybe exclude it or possibly suggest further lines of investigation - all leading towards a proper diagnosis for the patient. So the fact that a patient is being referred for a bone scan does not necessarily mean that they have cancer, although this may well be what is going through a patient's mind.

\section{Time out 4.}

Suppose that you are looking after a 55 year old male patient with known cancer of the prostate in whom secondary spread to the bones is suspected and they have been referred for a nuclear medicine bone scan. Thinking about your own questions in time out 1 and 2, write 
some notes about what you would say to the patient if they asked you why they were going to nuclear medicine and what would happen there.

\section{Myocardial perfusion scan}

The myocardial perfusion scan can show blood flow to the heart muscle, so it is often used to investigate patients with ischaemic heart disease. The study is usually carried out in two parts in order to compare myocardial blood flow with the patient at rest and during "stress" (either physical exercise or pharmacologically induced myocardial stress). A comparison of the rest and stress images can be used to detect ischaemia - areas of the myocardium where blood flow is not able to meet the increased demands of stress due to narrowed coronary arteries. There are several different methods that may be used to stress the heart, either by physical exercise on a bicycle or treadmill or by infusion of a suitable drug. The local nuclear medicine department will give specific instructions for patient preparation depending on the stress method that they wish to employ for each patient. Patient preparation may involve refraining from taking certain medicines and foods and drinks containing caffeine for a period before the appointment. It is important that this advice is followed otherwise the scan may not be able to proceed.

When the patient attends for the stress part of the scan the procedure will be explained to them and then they will be stressed under careful supervision by suitably qualified and experienced staff. When their heart is adequately 
stressed they will be given an intravenous injection of a radiopharmaceutical which concentrates in the myocardium in proportion to the local blood flow at maximum stress. Then the patient will be asked to rest for about one hour before the images can be taken. This is to allow time for the radiopharmaceutical to disappear from other parts of the body so that good pictures of the heart can be obtained.

After about one hour of rest a gamma camera will be used to take pictures of the radiopharmaceutical in the heart muscle. The patient will be asked to lie flat on their back on an imaging couch and they will need to raise one or both arms above their head in order to allow the gamma camera to come close to their chest and keep them there for about 20 minutes (figure 4). If the patient thinks that they will not be able to manage this then it should be discussed with nuclear medicine staff before the appointment. The gamma camera will rotate around the patient's chest taking SPECT images (as already described for the bone scan). Some additional images may also be taken using a low dose CT scanner attached to the gamma camera. These can be used to apply corrections to the gamma camera images which improves the accuracy of the test. A computer will reconstruct these images into cross sectional slices through the heart.

Sometimes this stress scan is sufficient to produce a definitive result, but in many cases it is necessary to compare it with a similar scan performed at rest. In this case the patient will be given another appointment to have a rest scan. This may be later the same day or on a different day. The rest scan is 
performed in the same way as the stress scan except that the patient will not be asked to exercise and instead the radiopharmaceutical is injected whilst they are resting.

Figure 5 shows an example of the results of a myocardial perfusion scan. The image shows several slices through the myocardium with the amount of radiopharmaceutical, representing myocardial perfusion, shown by the bright colours. The top row of images, taken during stress, shows an area of reduced perfusion in the myocardium (yellow arrow). The bottom row of images taken at rest shows that blood flow to this area normalises at rest. This appearance is typical of ischaemia and indicates that the patient could benefit from a revascularisation procedure to this area of the myocardium.

In other patients the result might show that blood flow is abnormal at both stress and rest. This indicates that the myocardium is dead (infarcted) and so a revascularisation procedure will not help. On the other hand, if blood flow is found to be normal even at stress, then significant coronary artery disease is unlikely and the patient will have a very low risk of a cardiac event in the future.

\section{Time out 5}

Follow this link to watch a video of a patient having a stress myocardial perfusion scan 
(https://www.youtube.com/watch?v=REkCgpa5bK0). Write some notes about what you might say to explain this to a patient.

\section{PET-CT scan for oncology}

PET stands for Positron Emission Tomography; it is a special sort of nuclear medicine scan that is most often used to investigate patients with cancer. That is the application which is described here, although PET scanning can also be used for a variety of other conditions that do not involve cancer at all.

Before attending for a PET scan the nuclear medicine department will give the patient information about how to prepare for the scan. This may involve refraining from strenuous exercise and not having anything to eat for a period before the appointment. It is important that the patient follows the advice given as it may affect the result of their scan. When they attend the nuclear medicine department the patient will be given a small intravenous injection of a radiopharmaceutical which mimics the action of glucose. This is labelled with a radioactive element fluorine-18 which can be detected by a PET scanner. They will then be asked to rest quietly without talking for about 1 hour. This gives time for the radiopharmaceutical to become concentrated in areas of high glucose metabolism. This can identify fast growing areas such as tumours which need lots of energy, but it can also show uptake in active muscles - which is why the patient needs to keep still and relax after the injection. If the patient has eaten recently (which includes intravenous or tube feeding) or if they are a poorly controlled diabetic with high blood sugar, then 
the radiopharmaceutical will have to compete with natural glucose, resulting in a poor quality scan and potentially erroneous results.

After a rest of about 1 hour a PET-CT scanner will be used to take pictures of the radiopharmaceutical distribution. The patient will be asked to lie flat on their back on an imaging couch which moves into a ring shaped detector (see figure 2). It looks a bit like a CT scanner or an MRI scanner, but it does not make any loud noises like an MRI scanner. The scanner will take a series of PET images of different parts of the body as the bed advances into the scanner, which takes about 20 minutes. It will also take some X-ray CT images of the same areas. The PET and CT images are then combined to give a fused PETCT image which gives the best diagnostic information. Figure 6 shows an example of a PET-CT scan in a patient with lung cancer. The middle image is the PET image where high glucose metabolism is shown in black. The primary tumour can be seen in the left lung (red arrow) but there are also several metastases (blue arrows). The left hand image is the corresponding CT and the right hand image is a fusion of the PET and CT with the PET shown as shades of red and orange.

The PET-CT images are very useful for cancer staging, determining treatment options and monitoring response to treatment and in some hospitals they can also be used in external beam radiotherapy planning. 


\section{Time out 6}

Follow this link to watch a video of a patient having a PET-CT scan (https://www.youtube.com/watch?v=433-HAi8BTw). Write some notes about what you might say to explain this to a patient.

\section{Radiation protection issues}

For most nuclear medicine procedures the radiopharmaceutical remaining in the patient will gradually decay away or be eliminated in their urine over a few hours. This means that patients who have recently had a nuclear medicine procedure are potentially sources of radiation which could affect other people nearby. Different amounts of radiation are used for each imaging procedure and the speed and route of biological elimination are different for each radiopharmaceutical, so the radiation protection issues are not the same for all procedures. For bone scans and PET scans a reasonably large amount of radioactivity is injected, so patients will be sources of radioactivity for a few hours. Also much of the radioactivity is excreted via the kidneys, making the patients' urine radioactive. This can create radiation protection issues for nurses on the ward.

\section{Time out 7}

Can you write down any radiation protection issues for you and/or your colleagues if a patient in their care attended for a bone scan? 
How do you think that you would deal with these issues if you came across them?

The risk to other people from standing close to a patient who has had a diagnostic nuclear medicine procedure is very small and it will not normally exceed the limits that are deemed to be safe for members of the public. Radiation exposure reduces rapidly with increasing distance so it is sensible to avoid unnecessary prolonged close contact with patients, but this does not preclude normal nursing care. For some diagnostic procedures patients may be advised to avoid prolonged contact with pregnant women or children for a short while. If this is the case the local nuclear medicine department will give specific instructions. If no specific instructions are given then normal nursing care may be followed, but it would be sensible for a nurse who is pregnant to avoid activities that require intensive bedside care of a patient for 24 hours after the procedure. There is not usually any need to restrict contact between nuclear medicine patients and other patients or visitors.

For some nuclear medicine procedures the patient's urine may be radioactive for about 24 hours after the scan, but this is only likely to be a significant hazard to nursing staff if it is accidentally spilled on their body or ingested. Therefore normal nursing procedures, such as wearing disposable gloves and aprons when dealing with patient's urine and washing hands afterwards, will be adequate protection. 
Nuclear medicine departments will often ask for a nurse to accompany their patients to the department for their scan, but pregnant nurses should not be assigned to these duties as they will not be allowed into the radiation areas within the department.

For therapeutic nuclear medicine procedures the amount of radiation used is much greater and so patients receiving therapy may need to be placed in a special isolation ward for a while. However this only takes place in a few specialised hospitals and ward staff there will receive specific training for dealing with these patients and so therapeutic procedures are not within the scope of this article.

\section{Further reading}

This article has described examples of three common nuclear medicine procedures, the bone scan, myocardial perfusion scan and the PET-CT scan. However, by administering other radiopharmaceuticals that concentrate in different parts of the body nuclear medicine can also be used for imaging many other organs and body systems; for example lungs, kidneys, thyroid, parathyroid and brain. If you have registered with e-Learning for Healthcare (see time out 3) then you can read more details of all these procedures in the eLfH programme 'Interpretation of Radiological Images', nuclear medicine module. This contains an introductory section which explain the principles of nuclear medicine and how the gamma camera is used to take images. A 
section about common applications describes the bone scan, lung scan, renography, thyroid \& parathyroid imaging, stressing for myocardial perfusion studies and myocardial perfusion imaging. The Positron Emission Tomography section explains how a PET scan is performed and describes oncology and some non-oncology (brain and cardiac FDG) studies. There is a final section covering sentinel node localisation and radionuclide therapy. These sessions are all written at a level that is intended to be suitable for nurses and other healthcare professionals who are not nuclear medicine specialists.

\section{Summary}

This article has explained how nuclear medicine takes images showing the pathology of patients' body systems by administering small amounts of radiopharmaceutical - chemicals with a radioactive label attached. These are detected externally by gamma cameras or PET scanners. The images obtained are complementary to those obtained from other imaging modalities because they show function rather than anatomy. Modern hybrid scanners can combine nuclear medicine and CT images to give the best of both modalities.

Bone scans can be used to investigate a wide range of bone conditions, not just cancer. Myocardial perfusion scans are used to investigate patients with suspected coronary artery disease. PET scans are most often used to investigate patients with known cancers. Patients who have had a diagnostic nuclear medicine scan will remain radioactive for a while after the test, but as 
long as simple precautions are followed they will not pose any significant risk to nursing staff. 


\section{References}

Brenner A I, Koshy J, Morey J, Lin C, and DiPoce J (2012) The Bone Scan. Seminars in Nuclear Medicine, 42(1): p. 11-26

Department of Health (2010) NHS Brand Guidelines, http://www.nhsidentity.nhs.uk/tools-and-resources/patient-information

IRMER (2000) The Ionising Radiation (Medical Exposures) Regulations 2000. SI 2000, No 1059. HMSO, London.

IRR (1999) The Ionising Radiation Regulations 1999. SI 1999, No 3232. HMSO, London.

MHRA (2012) Best Practice Guidance on Patient Information Leaflets, https://www.gov.uk/government/uploads/system/uploads/attachment_data/file/

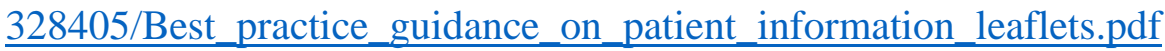

NHS Institute for Innovation and Improvement (2008)

http://www.institute.nhs.uk/quality_and_service_improvement_tools/quality_a nd_service_improvement_tools/patient_information.html 


\section{Figure captions}

Figure 1. A patient having a bone scan on a gamma camera

Figure 2. A patient having a positron emission tomography (PET) scan

Figure 3. Images from a normal bone scan.

Figure 4. A patient having a myocardial perfusion scan on a gamma camera

Figure 5. Images from a myocardial perfusion scan from a patient with ischaemia

Figure 6. Images from a PET-CT scan from a patient with lung cancer. 


\section{Multiple choice questions}

1. If you were asked to explain to a patient what a nuclear medicine scan might involve, which of the following statements are true?

a. For a bone scan the imaging only takes five minutes (False)

b. For a myocardial perfusion scan the patient has to lie still for approximately 20 minutes (True)

c. For a myocardial perfusion scan the patient may be required to avoid caffeine for a period of time prior to the scan (True)

d. For a PET-CT scan the patient should fast for 6 hours before the scan (True)

e. A PET-CT scanner makes a lot of noise (False)

2. For each of the following clinical conditions select an appropriate nuclear medicine imaging procedure from a choice of bone scan, myocardial perfusion scan or PET-CT scan

a. Suspected bone metastasis from prostate cancer (Bone scan)

b. Suspected coronary artery disease (Myocardial perfusion scan)

c. Staging of a patient with colon cancer (PET-CT scan)

d. Suspected hairline fracture of the scaphoid that is not seen on an x-ray (Bone scan) 
3. When a nuclear medicine imaging procedure is requested for a patient, which of the following statements are true?

a. The request must include adequate information to fully identify the patient for whom the test is required (True)

b. The requesting doctor or nurse must justify the fact that the benefit to the patient will outweigh the risk (False)

c. The requesting doctor or nurse must provide adequate information to the nuclear medicine department to allow them to justify the request (True)

d. Nuclear medicine imaging procedures cannot be carried out on pregnant patients (False)

e. The nuclear medicine department should be informed if the patient is breast feeding (True)

4. When looking after a ward patient who is having a myocardial perfusion scan, which of the following statements are true?

a. A nurse who is pregnant is not a suitable person to accompany the patient to the nuclear medicine department for their scan (True)

b. Nursing staff should keep more than 1 metre away from the patient for 24 hours after they have had their scan (False)

c. Nursing staff should wear disposable gloves when handling the patient's catheter bag (True)

d. A nurse who is breast feeding should avoid contact with the patient after their scan (False) 